\title{
THE STEINACH OPERATION: REPORT OF 22 CASES WITH ENDOCRINE INTERPRETATION
}

\section{HARRY BENJAMIN, M.D.}

NEW YORK

The ligation of the ductus deferens combined with vasectomy has become now generally known as the "Steinach operation." By its effects, I am inclined to designate it as "endocrine surgery." I shall assume that the theory on which Steinach based his practice is fairly well known, and discuss it only very briefly, referring to my other publications for more detailed information. $\dagger$

In the 22 cases herewith presented unilateral ligation under local anaesthesia was the rule. The vas was carefully isolated and separated from all accompanying structures for a distance of about $2 \mathrm{~cm}$. Two heavy silk ligatures were placed around the vas, about $2 \mathrm{~cm}$. apart. The one nearer to the testicle was reinforced by a fine silk ligature in order to use it later for sewing the lower stump into the tissue near the upper corner of the wound, for the purpose of suspension. Before this was done, a short piece of the vas between the heavy ligatures was resected. The tunica vaginalis and the skin were closed with catgut. The principle of the procedure is to close the vas, prevent re-communication of the stumps, and preserve the blood supply.

In a few cases I have observed irritation, and in two, even sloughing out of the silk without actual infection. Chromic catgut was therefore used in three cases, but Steinach and his surgeon strongly advise the use of silk, as it excludes the possibility of a re-opening of the vas. I am personally now using nothing else, but insist on double sterilization of the silk.

The place of the incision varied. I have either exposed the vas near the root of the penis or incised the scrotum and ligated near the epididymis. A somewhat quicker result can perhaps be looked for after the latter procedure. I have nowhere ligated between epididymis and testis.

†N. York M. J. [etc.]), 1922, 114, 687; Ibid., 1922, 116, 203; Am. M.ed. $1922,28,435$. 
According to Steinach's theory, ligation of the vas (not vasectomy alone) causes through back-pressure and later inactivity an atrophy of the generative portion of the testis, with subsequent proliferation of the so-called puberty-gland, consisting mainly of the interstitial (Leydig) cells. The increased formation of gonadal hormone re-activates the entire endocrine system of the patient, causing clinical results, varying from nothing more than an "endocrine tonic" to an almost actual "re-juvenation."

As an indication for the operation,-speaking generally and from an endocrine point of view-I have taken a hypofunction of the gonads, or better, an inferiority in the gonadal activity. I have assumed that "senility" is due to a naturally occurring, i. e., an acquired hypo-gonadism (primarily or secondarily) with an accompanying dysfunction of the rest of the incretory organs. If this state of affairs exists at a relatively early stage of life, I speak of "premature senility." Here, congenital inferiority of the gonads or the whole endocrine system plays perhaps a rôle. Such endocrine constitutional inferiority, or weakness of the gonads I also assumed in certain cases of sexual impotence (those of gonadal origin). Here the other endocrine organs are not affected in the same sense, and no symptoms of general senility are apparent. Those cases have also been described as "partial senility" and "partial premature senility," by Schmidt.

The principal contra-indications were: Advanced senility with atrophic testes, and a seriously impaired cardio-vascular apparatus. I have also refused the operation where it seemed that other therapeutic procedures, for instance, rest, should first be tried for symptoms of senility and where evidence of organic defects, like tabes, existed, to explain sexual impotence.

The important questions arise: Where can we expect fairly good results, where excellent ones, in what cases none at all, and can harm be done in ligating a man's vas, causing him to form a new puberty gland? The last question I am inclined to answer with "No." We have no proof whatever of any injurious effects, all the evidence pointing to the harmlessness of the procedure. Even where additional gonadal hormone is not required, we can trust in the balancing power of the endocrine apparatus as a whole. To the other questions I cannot give a definite answer, 
but would like to suggest a way of examination and classification which may in the future, and based on larger clinical material, enable us to understand more clearly the endocrinological consequences of the vasoligation, and thus differentiate favorable from unfavorable cases. I have therefore attempted to classify all cases according to their endocrinological "constitution," taking into consideration their life history, the character of their complaints, and their physical make-up. I have designated them as "thyroidal," "gonadal," etc., and where two glands seemed to be prominent in the patient's constitution, I described them as "thyro-gonadal," "pituito-adrenal," etc., putting the one most likely primarily delinquent in the first place. I have not differentiated between "hypo" and "hyper," unless this was glaringly justified.

I have interpreted the patient's symptoms from an endocrine point of view as far as possible, and likewise the changes that appeared after the operation. Then, taking the patient's constitution into consideration, I have sought to answer the question which constitution offers the best chances and in the sphere of which gland the principal changes might develop. This means clinically: Which are the favorable cases, and what will happen to them after the operation.

In view of the modernity of endocrine studies, a divergence of opinion might naturally arise regarding the classification of the constitution and the interpretation of symptoms. The study of more data is therefore imperative.*

The complaints of the senile cases were of the usual nature, partly mental and partly physical, and need not be described in detail.

The age in the 22 cases reported lay between 36 and 64 . They consist of (1) 12 cases of senility including premature and physiological old age (acquired gonadal deficiency); (2) 8 cases of congenital inferiority of gonads (hypogonadism) with sexual impotence; (3) 1 case of dyshormonism (No. 9); (4) 1 case of carcinoma.

Cases 1 to 8 have already been reported elsewhere with clinical details, as previously stated, but at that time I had not touched upon the endocrine aspect.

* It seems fair to state that my endocrine interpretations are based on the plan originated by Dr. Joseph Frankel. 
Regarding results, it must be noted that it sometimes takes six months, perhaps even more, for the full quota of clinical effects to develop. Positive improvements within this period give no definite clue as to their final intensity. I have found it practical to classify the results as follows: (1) Negative, if no changes at all occurred attributable to the operation, after a period of six months or more; (2) Subjectively positive and doubtful, if the patient stated improvements in his subjective sphere only, doubtful regarding endocrine interpretation. These cases have naturally a limited value as scientific evidence; and (3) Positive, if clear evidence, for instance objectively, of a beneficial effect was at hand, in some cases naturally only "partial."

ENDOCRINE OBSERVATIONS AND FURTHER STUDY OF EIGHT CASES, PREVIOUSLY REPORTED

\section{Case 1. Clerk-58 Years}

cocele.

Constitution: Adreno-gonadal.

Operation: February 2, 1921, in combination with varicocele (Dr. Reitzfeld). This patient did not know that the Steinach operation was performed.

Last Examination (Dr. Reitzfeld, five months p. o.): Distinct mental and physical improvement; gain in weight of twelve pounds; no change in sexual life; no endocrine study was attempted.

Result: Positive (auto-suggestion excluded).

\section{Case 2. Merchant-57 Years}

Diagnosis: Premature senility; chronic arthritis in both knees.

Constitution: Pituito-gonadal.

Operation: November 1, 1921 .

Last Examination (seven weeks p. o.): Marked functional improvement in his knees; general condition decidedly improved; sexual power increased; a distinct thyro-pituito-gonadal influence apparently manifest, but the time of observation too short to draw reliable conclusions.

Result: Subjectively positive (Doubtful).

\section{Case 3. College Professor--55 Years}

Diagnosis: Premature senility; prostatic hypertrophy with frequent micturition; affection of the internal ear (sclerosis) with increasing deafness.

Constitution: Pituito-gonadal.

Operation: December 28, 1921.

Last Examination (seven month p. o.): Marked improvement in every way; hearing much better as proved by objective methods (Dr. Max Heimann); prostate smaller, urination normal; weight increased by six pounds; blood pressure constantly somewhat lower, within normal limits; sex-life unchanged.

Endocrine interpretation is doubtful; thyroidal and pituitary activity is improved. The effect in the gonadal sphere seems to have influenced the prostate only. For the libido the cortex cerebri is too 
important and perhaps only influenced by the gonads when psychic factors are favorable. This was not the case here.

Result: Positive.

\section{Case 4. Manufacturer-48 Years}

Diagnosis: Premature senility; marked sexual impotence (not improved by transplantation of human (?) testicle or any other treatment).

Constitution: Adreno-gonadal. out).

Operation: February 15, 1922 (ligature had possibly sloughed

Last Examination (four months p. o.): General improvement physically and mentally; gain in weight ten pounds at least; sexual ability decidedly better but not yet normal; nightly emissions occurred first time in several years; increase in libido very doubtful.

Thyro-adreno-gonadal influence is apparent, but only in a moderate degree.

Result: Partially positive.

\section{CASE 5. LABORER-38 YeARS}

Diagnosis: Premature senility with sexual impotence; varicocele. Constitution: Thyro-adrenal.

Operation: February 20, 1922.

Last Examination (March 30, 1922) : No definite changes.

Result: Patient could not be reached for re-examination; negative, six weeks p. o. Case not classified.

\section{Case 6. Contractor-58 Years}

Diagnosis: Senility (premature).

Constitution: Adrenal.

Operation: February 28, 1922.

Last Examination (August 18, 1922, 51/2 months p. o.) : Marked improvement; more ambition and no more mental depression; gain of about six pounds in weight; appearance much better, complexion markedly improved; former frequent urination normal.

Patient says that he perspires more freely than in former years, and also noticed a stronger growth of his beard, as evidenced by necessity of more frequent shaving. His blood pressure (syst.) was around 180; is now 150 . Pulse pressure has decreased from 85 to 75 . His prostate showed a distinct hypertrophy, especially of the left lobe: It is still large, but both lobes alike, the left one having decreased in size. The skin reaction has changed from faintly red with white borders to a distinct red line of long standing. He feels perfectly able to resume business responsibilities which he had dropped for years. Sex desire and ability has increased little if any.

Here the changes in perspiration, complexion, growth of beard, blood-pressure and skin reaction indicate increased thyroid activity, balancing the (constitutional) adrenal dysfunction, as evidenced by blood pressure and skin reaction (Sergent line).

Result: Strongly positive.

\section{CASE 7. WATCHMAKER-36 YEARS}

Diagnosis: Sexual impotence for the last nine years; premature senility.

Constitution: Gonadal.

Operation: March 6,1922.

Last Examination (August 16, 1922, 51/2 months p. o.): Decided mental and physical improvement; patient has returned to work that he had discontinued for many months; feels as well and ambitious as ever; more self-assurance; acne on back disappeared.

The patient volunteered two interesting statements: First, he 
had been using a $21 / 2$-inch focus magnifying glass for his work which he finds too strong now; is using a $3 \frac{1}{2}$-inch focus now; second, has to shave every day compared with every two to three days formerly; he claims that his hair is growing "awfully fast." Sexual impotence is practically cured and he has had sexual intercourse satisfactorily for the first time in the last 9 years. He intends to get married.

Here also the thyroidal stimulation is evident, in addition to the increased gonadal activity in accordance with his constitution.

Result: Strongly positive.

\section{CASE 8. EDITOR-58 YeARS}

Diagnosis: Premature senility.

Constitution: Pituitary-adrenal.

Operation: March 15, 1922.

Last Examination (August 18, 1922; 5 months p. o.) : All physical and mental complaints have completely disappeared, especially his frequent (pituitary) headaches; enjoys perfect health; skin reaction has changed from red with white borders to distinctly red; (syst.) blood and pulse-pressure have dropped $10 \mathrm{~mm}$. within normal limit; sexual limitations are unchanged.

In accordance with the patient's constitution the balancing of the pituitary and adrenal dysfunction is very evident. This patient is a highly intelligent and critical man. No change in the gonadal sphere is apparent.

Result: Positive (except sexually).

For additional details as to symptoms and other clinical data of the above eight cases, I wish to refer to my previous publication of August 31st, 1922, in "Am. Med."

\section{PRELIMINARY REPORT OF 14 ADDITIONAL CASES}

Case 9. Engineer-36 Years (Referred by Drs. D. M. Kaplan and GREG. STRAGNELL)

Principal Complaints and Symptoms: Patient feels physically and mentally "inferior"; has frequent (pituitary) headaches and digestive disturbances; suffers from premature ejaculation and partial sexual impotence.

Diagnosis: Dyspituitarism with secondary dysgonadism; giantism; varicocele; scoliosis; chronic otitis media with moderate deafness.

Constitution: Pituitary.

Operation: March 17, 1922, in combination with partial resection of the varicocele (the Steinach operation was performed as an attempt to influence the endocrine system); a part of the silk ligature sloughed out without infection.

Last Examination (31/2 months p. o.) : After "ups and downs" doubtful as to resulting from operation, the patient now states that his headaches have decidedly improved in that they are less frequent and less intense; better digestion; sex desire irregular but more frequent nightly emissions; no opportunity to test his potency; no objective changes in weight, blood pressure, etc.

Result: Doubtful, subjectively positive (although the improvement suggests an influence on his constitutionally inferior pituitary).

Case 10. Dentist-39 Years. (REFerRed by DR. Strikler of PHILADELPHIA)

Principal Complaints and Symptoms: Sexual weakness began four years ago; now complete impotence; 25 years ago contracted syphilis that was treated until several Wassermann examinations of blood and one of spinal fluid were negative; knee jerks distinctly exaggerated, otherwise physical examination negative. 
Diagnosis: Impotentia sexualis somatica; psoriasis; lues (cured?).

Constitution: Adrenal.

Operation: March 17, 1922.

Report by letter: No change (21/2 months p. o.).

Result: So far negative.

\section{Case 11. AGENT-56 Years}

Principal Complaints and Symptoms: Fifteen years ago one kidney removed on account of stones; frequent attacks of dizziness; poor memory and lack of ambition; slight deafness; glycosuria, disappearing after a few days of strict diet.

Blood Pressure: (Syst.) between 160 and 170; pulse pressure between 60 and 80 ; weight, 138 pounds; no skin reaction; sex desire and ability gradually waning; prostate enlarged; occasional slight attacks of gout; irritable and depressed.

Diagnosis: Premature senility; beginning arteriosclerosis; diabetes mellitus.

Constitution: Thyro-adrenal.

Operation: March 27, 1922. (Healing of wound delayed.)

Last Examination (41/2 months p. o.): In the intervening time there were "ups and downs" in patient's subjective complaints; no improvement in his sugar tolerance and in his sexual limitation apparent; indigestion, probably due to his lack of teeth and inability to masticate properly; less depressed and somewhat more ambition; generally livelier, looks better and hears better as noticed by his rela. tives; his skin reaction, faintly red; his weight slightly increased (140 pounds); his attacks of dizziness practically ceased since about 4 weeks p. o.; his blood pressure dropped and now changes between 130 and 145, syst.; pulse pressure between 45 and 55 .

So far only slight stimulation of thyroidal activity seems to manifest itself, with subsequent effect on the adrenals.

Patient's improved hearing has been confirmed through objective methods by an ear specialist (Dr. Max Heimann).

Result: Partially positive.

\section{Case 12. Naval OFFICER-53 Years}

Diagnosis: Beginning arteriosclerosis; angina pectoris.

Constitution: Adreno-gonadal.

Principal Symptoms and Complaints: Mild but frequent attacks in heart region, radiating into left arm, occasionally quite severe; blood pressure, systolic, 155 ; diastolic, 110 ; pulse, 76 .

$\mathrm{X}$-ray examination of heart showed moderate enlargement of heart and aorta; weight $161 \frac{1 / 2}{2}$ pounds; sexual ability decreasing; prostate enlarged.

Operation: April 11, 1922; one month after the operation the subjective condition was about the same, but patient noticed stronger erections; his blood pressure, systolic, 135; diastolic, 90 ; weight, 163 pounds; otherwise no change.

Case not classified.

\section{CASE 13. IMPORTER-43 YEARS}

Diagnosis: Hypo-gonadism; sexual weakness.

Constitution: Gonado-pituitary.

Principal Complaints and Symptoms: Patient feels that through his sexual inferiority (weak desire as well as limited potency) he "is losing something in life"; desires marriage; fears premature senility. Operation: April 21, 1922 (ligation with chromic catgut).

Although no re-examination has been possible, the patient reported ( 5 months after operation) that no noticeable changes have occurred 
in his condition. He is in excellent health, physically and mentally. I am, however, inclined to classify, his case as

Result: So far negative.

\section{Case 14. Dentist-53 Years}

Diagnosis: Premature senility; beginning arteriosclerosis; impotentia sexualis; doubtful syphilitic history.

Constitution: Adrenal-gonadal.

Principal Symptoms and. Complaints: Nervous twitchings; in somnia; increasing weakness of sight and hearing; lack of "pep"; shortness of breath on exertion; sexual impotence for the last 10 years; x-ray examination of heart (Dr. Philips) shows a moderate general hypertrophy without insufficiency or dilation; blood pressure, systolic, around 140 ; pulse pressure, 60 ; pulse, $76-80$; weight, $155 \frac{1}{2}$ pounds.

Operation: April 29, 1922 (ligated bilaterally with chromic catgut).

Examination (one month p. o.): Patient claims to have more energy and ambition, but only since a few days; also distinctly better erections, but no intercourse attempted; blood pressure, systolic, 122; pulse pressure, 37; pulse, 64; weight, $1591 / 2$ pounds (4-pound gain). In a letter of July 31st from out of town, patient reported to be in about the same condition as on the last examination.

So far an influence on the adrenals and in the gonadal sphere is evident. Although objective changes seem to have occurred, the time of observation was too short to call the result so far other than doubtful.

Result: Doubtful.

\section{Case 15. Merchant-63 Years}

Diagnosis: Beginning senility.

Constitution: Pituito-gonadal.

Principal Symptoms and Complaints: Patient feels that oncoming of age interferes with his ability to work, especially in regard to memory, ambition, power of concentration, etc.; blood pressure, systolic, 138; pulse pressure, 58; prostate enlarged.

Operation: May 3,1922 (ligation with chromic catgut). (Traumatic non-infectious epididymitis followed, but healed promptly.)

A letter of August 5th (three months p. o.) reads in part: "... I am feeling fine since the operation. There has been a general improvement in my health, particularly the prostate trouble. I have gained some flesh. I cannot see that my sex powers have increased to any extent. ..."

(No endocrine interpretation attempted from written reports.)

Case not classified.

\section{Case 16. Electrician-39 Years}

Diagnosis: Premature senility; sexual impotence.

Constitution: Thyro-gonadal.

Principal Symptoms and Complaints: Mentally depressed, cannot keep a good position through lack of ambition; premature ejaculation amounting to sexual impotence that depresses him greatly; looks almost senile-about 15 years older than his age; organic mitral heart lesion well compensated; blood pressure, systolic, 142; pulse pressure, 32; weight, 136 pounds.

Operation: May 26, 1922.

Examination: June 5, 1922. Blood pressure, systolic, 115; pulse pressure, 30 ; weight, $1373 / 4$; no change of subjective symptoms. Since no further report has been received of this patient, his case has not been classified. 
Case 17. Clerk-55 Years

Diagnosis: Carcinoma of head of pancreas.

Constitution: Thyro-adrenal.

Principal Complaints and Symptoms: Pain in stomach and loss of weight; obstructive jaundice; blood pressure, systolic, 130; pulse pressure, 45 .

Operation: June 1, 1922.

Dr. Heckman performed laparotomy, found cancer inoperable, adherent to vena cava and aorta; metastasis in liver; bilateral Steinach operation near epididymis.

Last Examination, August 1, 1922 (two months p. o.) : The patient has had 6 x-ray treatments (Dr. Thos. Scholz); subjective complaints diminished; weight about the same as after the operation; blood pressure, systolic, 108; pulse pressure, 33 ; strength not decreased; feels "lots of physical and mental energy"; the intensity of the icterus changed, now about the same as shortly after the operation; patient left town.

A change in the size of the palpable tumor was not evident; however, his constitutional thyro-adrenal sphere shows certain signs of being influenced. The cancer-chachexia has certainly not increased in the two months after the operation.

At the end of September (4 months after the operation), according to his son's report, patient still holding his own; had been traveling a good deal; digestive disturbances distinctly improved; lost no more weight and felt quite satisfied.

Result: So far doubtful, perhaps partially positive.

Case 18. Physician-64 Years

Diagnosis: Beginning senility; arteriosclerosis.

Constitution: Adreno-gonadal.

Principal Symptoms and Complaints: Decreasing mental faculties; poorer memory; very irritable; increasing sexual weakness; blood pressure, systolic, 195; pulse pressure, 85; weight, 161; prostate slightly enlarged.

Operation: June 27, 1922; in a letter dated August 5th (about 5 weeks p. o.), the doctor writes: "... I began to improve every day after I left the hospital. Have gained in flesh and now weigh 172 in my light weight clothes. I feel that my manhood is very much improved and I took my blood pressure this morning, systolic, 175, and diastolic, 120. ...."

(His pulse pressure was 55, as compared to 85.) "Everybody tells me I am looking better than when I left for New York."

Considering that this patient is a physician himself, I am inclined to call the

Result: So far, partially positive.

\section{CASE 19. Pharmacist-38 Years}

Diagnosis: Impotentia sexualis somatica (hypo-gonadism).

Constitution: Gonado-(hypo)-adrenal.

Principal Symptoms and Complaints: Premature ejaculation with erections rare and considerably weaker than in former years; all treatments including organotherapy unsuccessful; happiness of his married life greatly endangered; mental depression; blood pressure, 140 ; pulse pressure, 60 ; weight, 159 pounds.

Operation: August 4, 1922.

Not classified.

CASE 20. DYer-39 Years

Diagnosis: Hypogonadism; impotentia sexualis.

Constitution: Thyro-gonadal (hypo-gonadal). 
Principal Symptoms and Complaints: Decreasing sexual ability with frequent mental depression; all treatments unsuccessful; right testis slightly atrophic through accident in childhood.

Operation: August 7, 1922.

Examination, August 19, 1922: Patient had a pollution two nights ago, first in many months; is sure that erections are stronger; blood pressure, systolic, 110, compared to 122 before operation; pulse pressure, 40 , compared to 42 ; no other change.

Here ten days after the operation, a greater activity in his constitionally inferior gonadal sphere seems already to manifest itself.

Last Examination, September 16 (5 weeks after operation) : Distinct improvement; unusual sense of well-being; "rheumatic" pains in knees disappeared; pulse, blood pressure, and skin reaction normal as before operation; can read without glasses much easier than before; sexually, a marked improvement; erections are a good deal better; ejaculation still somewhat premature, but more pleasurable.

The improvement of gonadal activity is still more apparent and since the patient does not belong to the class of psychopaths I would already call the

Result: Positive.

\section{CASE 21. PATROLMAN-45 YearS}

Diagnosis: Hypo-gonadism; sexual impotence.

Constitution: Gonad-(hypo)-thyroidal.

Principal Symptoms and Complaints: Decline of sexual ability for the past seven years; all treatments unsuccessful; testes somewhat undersized; skin reaction, white line.

Operation: August 8, 1922.

Last Examination, September 26 (6 weeks p. o.) : Patient feels generally unusually well, but has so far not noticed any increase in sexual powers, except for a few days immediately after the operation; nervous temperament still the same; temporary decrease of blood pressure, then return to normal; skin reaction changed into a red line with slightly white borders; gained about 6 pounds in weight.

Increased thyroidal activity seems very probable, but in the gonadal sphere no changes have occurred, therefore,

Result: So far negative.

\section{Case 22. Music Teacher-54 Years}

Diagnosis: Beginning senility.

Constitution: Thyroidal.

Principal Symptoms and Complaints: Decline in mental and physical capacities for past 4 years; endurance, concentration and memory weakened; position endangered; was asked to resign on account of advancing age; frequent úrination; prostate enlarged; blood pressure, systolic, 155; pulse pressure, 50; weight, $155 \frac{1 / 2}{2}$ pounds; sexual ability decreasing.

Operation: August 8, 1922.

In a letter of September 1 ( 3 weeks after the operation) patient reports very hopefully, emphasizing especially a distinct improvement in urination, the urine flowing "with a much freer and fuller flow than it has for years"; a distinct increase in frequency and strength of erections also noted. No re-examination has so far been possible. I have, therefore, not classified this case at all.

\section{CONCLUSIONS AND SUMMARY}

Although being far from having arrived at definite conclusions, I believe that certain observations are sufficiently suggestive to form at least a plausible working basis. Of the 22 cases, 
6 were not classified, since no report was received about their condition. The results, therefore, can only be judged in 16 cases. Of these, I consider 9 positive (more or less strongly); 4 doubtful or subjectively positive; 3 negative. The doubtful and negative cases may have to be judged differently after longer observation.

Regarding the endocrine manifestations, I would like to summarize my observations as follows, giving a theory more than scientific facts.

The most frequent and most prominent clinical manifestations as the result of the new activity of the puberty glands seem to be due to increased thyroid activity.

Outside of the thyroid, the re-activated puberty gland seems to influence essentially those glands that are most prominent or delinquent in the patient's constitution.

Distinct changes in the gonadal sphere appeared mostly in patients with a gonadal or partly gonadal constitution. Therefore, the claim of certain German investigators that the increase of sexual activity was regularly observed could not be fully substantiated.

The following, however, must be.considered as well established scientific facts, also deduced from the above observations.

The Steinach operation increases the activity of the puberty gland. This re-activation has an influence on the endocrine system in the sense of stimulation, regeneration, and restoration of equilibrium. No injurious effects have been observed.

Regarding prognosis, I can at present express only a very vague opinion that gonadal or partly gonadal constitution seem to be most promising. I believe that a further close observation of the treated cases from an endocrine point of view will greatly enhance our knowledge of the inter-relationship of the different glands, especially the inter-relationship of the gonads with the rest of the endocrines. 